\title{
Brucella canis EN MEDELLÍN (COLOMBIA), UN PROBLEMA ACTUAL
}

\section{Brucella canis IN MEDELLÍN (COLOMBIA), A CURRENT PROBLEM}

\author{
Carlos Andrés Giraldo Echeverri ${ }^{1}$ \\ Zulma Tatiana Ruiz Cortés ${ }^{2}$ \\ Martha Olivera Ánge $\left.\right|^{3}$
}

\section{RESUMEN}

La brucelosis canina tiene impacto en la producción canina y en la salud pública, a nivel mundial, ya que genera pérdidas económicas a los criadores por infertilidad y por abortos, y existe el riesgo de transmisión zoonótica a los trabajadores y a los propietarios de mascotas. En Colombia, se conoce muy poco acerca de la frecuencia de presentación de la enfermedad, además, no existen leyes sanitarias que la regulen. En el laboratorio Syngamia de la Universidad de Antioquia, se ofrece el diagnóstico desde 2005, con la prueba tamiz de aglutinación rápida, en placa con $2 \beta$-mercaptoetanol y hemocultivo confirmatorio. A partir de los resultados obtenidos de los pacientes remitidos a este laboratorio, se halló una frecuencia de presentación del $11 \%$ de seropositividad, en el ámbito regional. Al analizar las historias clínicas, se encontró, por una parte, que los propietarios consultan muy poco al Médico Veterinario, y aquellos que consultan encuentran una diversidad de criterios sobre las acciones a seguir, cuando el criadero o la mascota

${ }^{1}$ M.V., MSc. Grupo Vericel, Facultad de Ciencias Agrarias, Universidad de Antioquia. Carrera 75 \# 65-87, Medellín, Colombia. Autor para correspondencia. e-mail: cargiraldo@gmail.com.

2 M.V., MSc, PhD Grupo Biogénesis, Facultad de Ciencias Agrarias, Universidad de Antioquia. e-mail: ztatiana@ gmail.com.

3 M.V., MSc, Doctora en Ciencias Agrarias, Grupo Vericel. e-mail:syngamia@gmail.com. resultan positivos a la enfermedad. El objetivo de este estudio es mostrar la casuística de tres años de servicio diagnóstico y las aproximaciones que se propusieron para el posible control de la enfermedad.

Palabras clave: Brucelosis, criadero perros, urbano, zoonosis.

\section{SUMMARY}

Canine brucellosis has impact on kennels and worldwide public health, generating economic losses for breeders caused by infertility and abortions, and potentiating the risk of zoonotic transmission by pets and workers. In Colombia, little is known about the frequency of the disease and there are no regulations for control or prevention of the illness in animals or humans. At the Universidad de Antioquia in the "Syngamia" Laboratory the diagnosis is offered since 2005, with the agglutination probe with $2 \beta$-mercaptoetanol and hemoculture for confirmation of the positives samples. Taking into account the results obtained in the lab, the frequency of seropositivity is $11 \%$ in the region; when the histories were analyzed it was found that breeders and pet owners do not use the veterinary service, and those that attend the clinic received a diversity of criteria about what to do when animals are positive. The objective of this study was to show the results of three years casuistic in our lab and to propose a protocol for control of the illness based on our experience and laws of other countries legislation.

Key words: Brucellosis, kennel, urban, zoonosis. 


\section{INTRODUCCIÓN}

La industria canina en Colombia muestra un crecimiento del $5 \%$ anual en los últimos cinco años, con una población aproximada de 4.500 .000 perros, incluidos los criaderos y los animales de compañía (información personal Purina S.A.). Este crecimiento trae consigo el aumento de problemas sanitarios, como la Brucelosis canina, lo cual, es un inconveniente de salud pública, por su potencial zoonótico. Aunque en el mundo solamente se hayan reportado alrededor de 40 casos de humanos positivos con aislamiento de Brucella canis (Olivera E Di-Lorenzo, 2009; Baldi et al. 1996) y, recientemente, en Argentina, se haya realizado un estudio, mediante ELISA, mostrando una baja incidencia (Lucero et al. 2005); a nivel de la ciudad de Medellín (Antioquia), se realizó el aislamiento de la bacteria de una persona conviviente con animales enfermos, sin que presente sintomatología de la enfermedad (Di Lorenzo \& Olivera, 2008); sin embargo, el diagnóstico de la zoonosis por $B$. canis en humanos, a nivel mundial, ha sido difícil de estimar, debido a la falta general de pruebas serológicas, a las reacciones cruzadas con otras especies de Brucella spp., y a las ideas erróneas de su prevalencia (Lucero et al. 2005).

La Brucelosis canina está ampliamente distribuida en el mundo y se comporta, epidemiológicamente, como la enfermedad reproductiva más relevante en esta especie (Wanke, 2004; Carmichael E Shin, 1996; Myers \& VarelaDíaz, 1980). B. canis fue aislada por Carmichael en 1967, quien la identificó como un pequeño cocobacilo intracelular gram-negativo, que forma colonias rugosas en cultivo, crece en agar tripticasa soya y no requiere mantenimiento en $\mathrm{CO}_{2}$; aún así, una tasa de prevalencia mundial para esta enfermedad, no se conoce aún (Shin \& Carmichael, 1999), a excepción de estudios en diferentes regiones (Ramírez et al. 2006; Marassi et al. 2003; Castillo et al. 2002; Almeida et al. 2004).

Los síntomas agudos de la brucelosis canina cursan con fiebre y decaimiento; sin embargo, son mas notorios los síntomas relacionados con el tracto reproductivo (Wanke, 2004). En un 75\% de las hembras infectadas, ocurre aborto entre el día 30 y el día 55 de gestación. Los cachorros abortados, se observan parcialmente autolisados, con edema y hemorragias subcutáneas en la región abdominal; luego del aborto, se presenta una descarga vaginal verde grisácea o marrón durante varios días (Borie Polanco, 2005), que es altamente contaminante (Keid et al. 2007). Los abortos pueden ser recurrentes o pueden parir cachorros vivos, que mueren pocas horas después del nacimiento (Charmichael $\mathcal{E}$ Kenney, 1968); además, la leche de estas perras es una fuente de eliminación de brucelas (Di Lorenzo \& Olivera $M, 2008)$. Los cachorros, aparentemente sanos, pueden desarrollar la enfermedad con el tiempo (Nicoletti $\varepsilon$ Chase, 1987), o presentar el tipo congénito de la enfermedad, que cursa con discoespondilitis (Kerwin et al. 1992) y uveítis anterior (Saegusa et al. 1977). Otro de los síntomas en los criaderos es la pérdida embrionaria entre los diez y los 20 días pos-servicio, que se interpreta como "servicios no efectivos" (Shin E Carmichael, 1999).

En el macho, durante la fase aguda de la infección, los síntomas clínicos más frecuentes son epididimitis y prostatitis, que conducen a impotentia generandi. Debido al dolor que produce la inflamación, el perro se lame el escroto con frecuencia, lo que conlleva a que desarrolle una dermatitis que, comúnmente, se contamina con estafilococos no hemolíticos. En los primeros tres meses pos-infección, el cuadro espermático de machos infectados muestra teratospermia, con abundantes células inflamatorias (Shin $E$ Carmichael, 1999). La fase crónica de la enfermedad cursa con atrofia del epidídimo, que termina en atrofia testicular (Wanke, 2004; Schoeb E Morton, 1978), con la consecuente azospermia (Shin E Carmichael, 1999). En el plasma seminal, se pueden encontrar anticuerpos antiespermatozoides (Serikawa et al. 1984).

En los criaderos, los problemas asociados a la reproducción (abortos, nacimientos prematuros -menos de una semana de la fecha prevista- y servicios no efectivos), se ha convertido en un problema de consulta frecuente entre los veterinarios clínicos (Jara et al. 2005; Flores-Castro et al. 1977).

El diagnóstico que se realiza en el laboratorio Syngamia de la Universidad de Antioquia es a partir de una prueba tamiz de aglutinación rápida en placa, con la cepa $M(-)$, añadiendo $2 \beta$-mercaptoetanol (Myers $\mathcal{E}$ Varela-Díaz, 1980); además, se lleva a cabo la prueba de inmunofluorecencia indirecta (Mateu-de-Antonio et al. 1993), que detecta infecciones mas tempranas. La prueba de oro para confirmar la presencia de $B$. canis es el aislamiento, posterior a un hemocultivo (Alton 
GG et al. 1988). El área de cobertura diagnóstica de este laboratorio corresponde al Área Metropolitana del Municipio de Medellín, aunque, ocasionalmente, se procesan muestras de otro destino departamental y nacional.

El objetivo de este estudio fue el de mostrar los resultados de las serologías que se realizaron en el laboratorio, en muestras remitidas por los veterinarios particulares, por veterinarios asesores de criaderos y por particulares, entre 2005 - 2007 y proponer algunas medidas de control.

\section{MATERIALES Y MÉTODOS}

Este estudio es de tipo retrospectivo, con la finalidad de medir la frecuencia de presentación de seropositividad a $B$. canis, en caninos de la ciudad de Medellín y alrededores, entre 2005 y 2007.

Muestras procesadas: Para este estudio, no se realizó un muestreo de la población; se procesaron 1.467 sueros remitidos al Laboratorio Syngamia, (Facultad de Ciencias Agrarias, Universidad de Antioquia), que procedían de criaderos caninos locales (Área Metropolitana, Antioquia), de pacientes llevados por el propietario al Hospital Veterinario de la Universidad de Antioquia, o de clínicas veterinarias particulares. Las muestras ingresaron con una nota de remisión, la cual, incluía la siguiente información: nombre propietario, teléfono de contacto, nombre del paciente, raza, edad, sexo y el motivo por el cual se solicitaba la prueba diagnóstica, si era remitido por un veterinario, un criadero rural o urbano y si era animal de compañía; en caso de ser de criadero, indicar cuantos animales habían en el mismo. En algunos casos, esta información fue incompleta, por omisión de datos en la nota de remisión.

Métodos diagnósticos: El diagnóstico tamiz de seropositividad, se ejecutó con la prueba de aglutinación rápida en placa, con la cepa $M(-)$ de $B$. canis, adicionando $2-\beta$-mercaptoetanol (2ME-PARP): se mezclan $25 \mu \mathrm{Ldel}$ suero, $25 \mu \mathrm{L}$ de $2-\beta$-mercaptoetanol y $50 \mu \mathrm{L}$ de antígeno. Se esperan hasta dos minutos observando las muestras en un aglutinoscopio, determinando como positivas aquellas que presentan aglutinación fina, similar al control positivo (Carmichael $\mathcal{E}$ Joubert, 1987). El antígeno es procesado en el laboratorio Syngamia, a un volumen celular de 6 a $8 \%$ de la cepa cultivada, cosechada e inactivada, con un $\mathrm{pH}$ de 7,4 (Carmichael E Joubert, 1987).

A todos los pacientes positivos a 2ME-RSAT, se les solicitó una nueva muestra de sangre completa para realizar hemocultivo; no en todos los casos fue posible obtener esta segunda muestra. En muestra de sangre entera, se colectó inicialmente, en agar sangre; luego, se siembra en agar tripticasa soya y se cultiva a $37^{\circ} \mathrm{C}$, por 48 horas (Carmichael $\&$ Joubert, 1987). Todos los aislamientos de los colonias que crecieron, se enviaron para certificación al Instituto Nacional de Referencia ANLIS "Doctor Carlos G. Malbran" (Buenos Aires, Argentina), en donde fueron confirmados para $B$. canis.

Análisis estadístico: Cuatro variables de la historia clínica (raza, sexo, edad y remitente) y los resultados de 2 ME-RSAT (positivo o negativo) fueron procesados usando el programa SPSS (ver. 14,0), para establecer las frecuencias específicas de cada una y las asociaciones de estas variables con el resultado de la serología, mediante la prueba chi-cuadrado $(\mathrm{p}<0,95)$.

\section{RESULTADOS Y DISCUSIÓN}

Las frecuencias de seropositividad y seronegatividad a $B$. canis, según el remitente de la muestra, fueron: clínica veterinaria, 15\% (14/93); propietario 9,1\% (60/657) y veterinario de criadero $8,9 \%$ (64/717). No se encontró una correlación entre la seropositividad y quien la remite. Al revisar las notas de remisión, se determinó que la mayor parte de los servicios se hacen por monta natural, los reproductores se prestan o se alquilan entre los criadores, que la mayoría de los criaderos son urbanos y que no contaban con asesoría profesional en los aspectos de salud, prevención de enfermedades y manejo genético (datos no mostrados). Los datos incompletos de los pacientes no permiten concluir acerca de la sintomatología relacionada con los seropositivos; sin embargo, el mayor porcentaje $(48,9 \%)$ de las muestras remitidas (1.467) fue de criaderos, donde se habían reportado casos de abortos en el último tercio de la gestación, problemas de infertilidad de hembras o machos o por requerimiento del diagnóstico para servicio reproductivo por monta directa o inseminación artificial. Esto, posiblemente, se debe a las condiciones técnicas y de manejo reproductivo de los criaderos, anteriormente mencionadas, donde, principalmente, los servicios reproductivos se hacen por monta natural 
y donde los perros seronegativos comparten espacios comunes con los seropositivos, aumentando el riesgo de infección, al estar en contacto estrecho con secreciones vaginales, prepuciales, sangre, orina y heces (Ramírez et al. 2006; Castillo et al. 2002; Carmichael E Shin, 1999).

Al igual que otros estudios de prevalencia de B. canis (Ramírez et al. 2006; Castillo et al. 2002; Almeida et al. 2004; Megid et al. 1999; Carmichael E Shin, 1999), no fue posible encontrar una asociación entre la raza y el diagnóstico sexológico; no obstante, se hallaron unas frecuencias de seropositividad importante, entre el 8,5 y el $17 \%$, entre las principales razas de criadero (bulldog, poodle, pug, beagle, schnauzer, schitzú, labrador retriever, maltés) y, por lo tanto, mascotas, a nivel local.

Aunque se considera que la edad no es un factor predisponente a la enfermedad y que los caninos se pueden infectar en cualquier momento (Carmichael $\mathcal{E}$ Shin, 1999), se asoció esta variable con el resultado del 2ME-RSAT, pero no fue posible una clasificación precisa por edad de los animales, ya que los propietarios y los criadores no llevan un registro adecuado de la fecha de nacimiento de sus animales, por lo tanto, las edades estimadas, se agruparon en rangos. No se encontró una asociación entre la seropositividad o seronegatividad con el rango de edad del animal, lo cual, es similar a los hallazgos de otros estudios (Ramírez et al. 2006; Castillo et al. 2002; Almeida et al. 2004; Megid et al. 1999; Carmichael E Shin, 1999); sin embargo, se encontró un mayor número de muestras remitidas y mayor seropositividad, en los caninos hasta los tres años de edad, posiblemente, debido a que al ser jóvenes tienen una mayor actividad reproductiva, por lo tanto, los propietarios desean conocer el diagnóstico en este rango de edad, para comenzar a cruzarlos, y, a su vez, tienen mayor riesgo de infección.
Respecto a la frecuencia de presentación de la enfermedad, según el sexo, se obtuvieron resultados similares entre machos y hembras $(10,9 \%$ hembras, $8 \%$ machos), sin una asociación significativa. En aparencia, no hay una predisposición por sexo y, por su carácter de transmisión venérea, no parece haber una discriminación de este tipo. Para este estudio, el número de muestras procesadas fue superior para las hembras, ya que son la población más común en los criaderos y por la remisión de muestras por parte de veterinarios con pacientes con síntomas de aborto, mucho más evidente, que los síntomas de infertilidad en machos.

Como se muestra en la tabla 1 , el $11 \%$ de la muestras fueron positivas a la prueba $2 \mathrm{ME}-\mathrm{RSAT}$, la cual, es una frecuencia de presentación de esta enfermedad más baja, comparada con estudios similares, realizados en Bogotá (Colombia), con 20,3\% de seropositivos (Castillo et al. 2002). En el ámbito mundial, se han encontrado otras frecuencias de presentación de la enfermedad, usando la técnica de inmunodifusión en agar, de la siguiente manera: en dos provincias de Callao (Perú), con 15,6\% de seropositivos (Ramírez et al. 2006); en la ciudad de Alfenas (Brasil), con 14,2\% (Almeida et al. 2004) y en Rio de Janeiro (Brasil), con 7,4\% de seropositivos (Marassi et al. 2003).

A todos los remitentes de perros positivos a 2ME-PARP, se les solicitó una muestra de sangre entera, con el propósito de realizar un hemocultivo. Solamente, se logró recolectar 40 muestras, de las cuales, se aisló la bacteria en 16 de los hemocultivos (Tabla 1). Dos de los reproductores machos positivos a hemocultivo fueron perros recientemente introducidos al país, como animales de compañía que, a su vez, sirvieron de reproductores. La legislación sanitaria colombiana (Instituto Colombiano Agropecuario, 2004 y 2003), para ingreso y egreso de caninos como animales de compañía, o para importación o exportación de reproductores, no

Tabla 1. Sensibilidad y especificidad de la Resultados de 2ME-RSAT y hemocultivos.

\begin{tabular}{|c|c|c|}
\hline Prueba $(\boldsymbol{n})$ & Positivo $(\boldsymbol{n})$ & Negativo $(\boldsymbol{n})$ \\
\hline 2ME-RSAT $(1467)$ & $11 \%(166)$ & $89 \%(1301)$ \\
\hline Hemocultivo $(40)$ & $40 \%(16)$ & $60 \%(24)$ \\
\hline
\end{tabular}


obliga a que los mismos sean negativos a B.canis. El hemocultivo es la prueba de oro en B.canis. ; sin embargo, su sensibilidad es baja, además que requiere un laboratorio de bioseguridad tres, para realizar esta prueba, así que se consideró que la $2 \mathrm{ME}-\mathrm{RSAT}$ es una prueba de selección rápida y práctica para la región en estudio, para tomar decisiones sobre manejo sanitario en el criadero, al igual que Reisz (2006).

Como estrategia de control, se indicó a los Médicos Veterinarios el parámetro que sugieren Shin \& Carmichael (1999): dos pruebas 2ME-RSAT consecutivas, con intervalo de 20 días, hemocultivo de los seropositivos y sacrificio para todos los perros positivos.

El contacto estrecho entre las personas y los perros infectados aumenta el riesgo de transmisión; aún así, su impacto sobre la salud pública es probablemente subestimado, debido a la falta de presentación de informes científicos y a la insuficiencia de servicios de diagnóstico (Zamora et al. 1980). El grupo de investigación Vericel fue el primero en reportar en Colombia un aislamiento de $B$. canis de una persona conviviente con un criadero infectado, sin sintomatología clínica, pero este hallazgo muestra que, posiblemente, se estaría subdiagnosticando el contagio con $B$. canis, como problema de salud pública urbana (Baldi et al. 1996).

Con base en los resultados obtenidos, se sugiere la siguiente estrategia de control de esta enfermedad: (a) los caninos deben tener una identificación clara; (b) durante el proestro realizar 2ME-PARP a todas las hembras que van a ser servidas; (c) repetir una semana antes del servicio; (d) todos los reproductores machos deben tener 2ME-PARP, con resultado negativo, al menos dos veces por año si son usados como donantes de semen para inseminación artificial; (e) todos los machos que se usen en monta directa para el servicio deben tener, al menos, un examen negativo 20 días antes del servicio y el día anterior del servicio; (f) se debe recomendar a todos los propietarios, que desean reproducir, que solamente usen inseminación artificial, sin contacto entre el macho y la hembra; ( $g$ ) cuando se introduce un animal nuevo a un criadero cerrado, éste debe permanecer en cuarentena al menos 30 días y practicar dos exámenes séricos durante este periodo, con 20 días de diferencia; (h) se recomienda el sacrificio de los animales 2ME-PARP positivos; (i) cuando los animales salen a competencias de agilidad o exposiciones caninas, se debe hacer examen antes de ingresar al lugar y 20 días después de terminada la competencia y, finalmente, (j) en caso de criaderos positivos, se recomienda hacer 2ME-PARP a las personas de las unidades familiares que permanecen en contacto con los animales y, en caso de resultar positivos, remitirlos a los médicos expertos en zoonosis.

\section{BIBLIOGRAFÍA}

ALMEIDA, A.C.; SANTORELLI, A.; BRUZADELLI, R. 2004. Soroepidemiologia da Brucelose canina causada por Brucella canis e Brucella abortus na cidade de Alfenas, Brazil. Arq. Bras. Med. Vet. Zoo. 56:275-276.

ALTON, G.G.; JONES, L.M.; ANGUS, R.D.; VERGER, J.M. 1988. Techniques for the brucellosis laboratory (Techniques et pratiques). Institut National de la Recherche Agronomique (INRA) Publications, Paris, France. p.169-174.

BALDI, P.C.; MIGUEL, S.E.; FOSSATI, C.A.; WALLACH, J.C: 1996. Serological Follow-up of human brucellosis by measuring IgG antibodies to lipopolysaccharide and cytoplasmic proteins of Brucella species. Clinical Infectious Diseases (Estados Unidos). 22:446-455.

BORIE-POLANCO, C. 2005. Infertilidad canina por Brucella canis. En: Olivera, M.; Gobelo, C. (eds.) El Libro Latinoamericano de Reproducción canina y felina. Ed. Biogénesis (Colombia). p.249-265.

CARMICHAEL, L.E.; SHIN, S.J. 1999. Brucelosis canina causada por Brucella canis. Am. J. Vet. Res. 37:220-223.

CARMICHAEL, L.E.; SHIN, S.J. 1996. Canine brucellosis: a diagnostician's dilemma. Semin Vet Med Surg (Small Anim.) (Estados Unidos). 11(3):161-165.

CARMICHAEL, L.E.; JOUBERT, J.C. 1987. A rapid slide agglutination test for the serodiagnosis of Brucella canis infection that employs a variant (M-) organism as antigen. Cornell Vet. (Estados Unidos). 77(1):3-12. 
CARMICHAEL, L.E.; KENNEY, R.M. 1968. Canine abortion caused by Brucella canis. J. Am. Vet. Assoc. 152:605-616.

CARMICHAEL, L.E. 1967. Canine brucellosis: isolation, diagnosis, transmission. Proc. Annu. Meet. U. S. Anim. Health Assoc. 71:517-527.

CASTILLO, V.; COTRINO, V.; MORENO, C. 2002. Encuesta serólogica sobre Brucella canis en pacientes atendidos en la clínica de pequeños animales de la Facultad de Medicina Veterinaria y de Zootecnia de la Universidad Nacional de Colombia (Sede Bogotá). Arch. Med. Vet. (Chile) 13:22-25.

DI LORENZO, C.; OLIVERA, M. 2008. Aislamiento de Brucella canis de leche de hembra canina infectada crónicamente. En: XXI Congreso panamericano de Ciencias Veterinarias (PANVET), Guadalajara, México.

FLORES-CASTRO, R.; SUÁREZ, F.; RAMÍREZ-PFEIFFER, C.; CARMICHAEL, L.E. 1977. Canine brucellosis: bacteriological and serological investigation of naturally infected dogs in Mexico City. J. Clin. Microbiol. (Estados Unidos). 6(6):591-597.

INSTITUTO COLOMBIANO AGROPECUARIO. 2004. Resolución No. 003336, 28 de diciembre de 2004. Disponible desde internet en: http://www.ica.gov. co/getattachment/1620253f-caae-4546-bdb1f1f598ace18e/3336.aspx (con acceso 10/12/08).

INSTITUTO COLOMBIANO AGROPECUARIO. 2003. Resolución No. 003382, 24 de noviembre de 2003. Disponible desde internet en: http://www. ica.gov.co/getattachment/fe51aa3f-8ef4-427b9f85-5731d95670fe/2003R3382.aspx (con acceso 10/12/08)

JARA, S.; PÉREZ, O.; DI LORENZO, C.; OLIVERA, M. 2005. Prevalencia de Brucella canis en una población canina de Medellín, Colombia. En: $12^{\circ}$ simposio internacional de la asociación mundial de laboratorios de diagnóstico veterinario, Montevideo.

KEID, L.B.; SOARES, R.M.; VASCONCELLOS, S.A.; CHIEBAO, D.P.; SALGADO, V.R.; MEGID, J.;
RICHTZENHAIN, L.J. 2007. A polymerase chain reaction for detection of Brucella canis in vaginal swabs of naturally infected bitches. Theriogenology (Estados Unidos). 68(9):1260-1270.

KERWIN, S.C.; LEWIS, D.D.; HRIBERNIK, T.N.; PARTINGTON, B.; HOSGOOD, G.; EILTS, B.E. 1992. Diskospondylitis associated with Brucella canis infection in dogs: 14 cases (1980-1991). J. Am. Vet. Med. Assoc. 201:1253-1257.

LUCERO, N.E.; ESCOBAR, G.I.; AYALA, S.M.; JACOB, N. 2005. Diagnosis of human brucellosis caused by Brucella canis. J. Medical Microbiology (Inglaterra). 54:457-461.

MARASSI, C.D.; MORAES, I.A.; LILENBAUM, W. 2003. Soroprevalência de brucelose canina no município do Rio de Janeiro pelo método de imunodifusão em gel agarose. Rev. Bras. Ciência Vet. 10(1):6364.

MATEU-DE-ANTONIO, E.M.; MARTÍN, M.; SOLER, M. 1993. Use of indirect enzyme-linked immunosorbent assay with hot saline solution extracts of a variant (M-) strain of Brucella canis for diagnosis of brucellosis in dogs. Am. J. Vet. Res. 54(7):1043-1046.

MEGID, J.; BRITTO, A.; MORAES, C.; FAVA, N.; AGOTTANI, J. 1999. Epidemiological assessment of canine brucellosis. Arq. Bras. Med. Vet. Zoo. 51: 94-98.

MYERS, D.M.; VARELA-DÍAZ, V.M. 1980. Serological and bacteriological detection of Brucella canis infection of stray dogs in Moreno, Argentina. Cornell Vet. (Estados Unidos).70(3):258-65.

NICOLETTI, P.; CHASE, A. 1987. An evaluation of methods to diagnose Brucella canis infection in dogs. Compend. Contin. Educ. Vet. (Estados Unidos) 9:1071-1073.

OLIVERA, M.; DI-LORENZO, C. 2009. Aislamiento de Brucella canis en un humano conviviente con caninos infectados. Reporte de un caso. Revista Colombia Médica. En prensa. 
RAMÍREZ, H.; CALLE, S., ECHEVARRÍA, L.; MORALES, S. 2006. Prevalencia de brucelosis canina en dos distritos de la provincia constitucional del callao. Rev. Inv. Vet. Perú. 17(1):39-43

REISZ, V. 2006. Evaluation of Bacteriological Cultures and Rapid Slide Agglutination Test with and without 2Mercaptoethanol in diagnosis of canine brucellosis. Master Degree. Disponible desde internet en: http://www.fmvz.unesp.br/eng/postgraduate/ VetMed/Dissertations_Theses/2006/pg_vm_an_ health_m.php (con acceso 10/07/08).

SAEGUSA, J.; UEDA, K.; GOTO, Y.; FUJIWARA, K. 1977. Ocular lesions in experimental canine brucellosis. Japan. J. Vet. Sci. 39:181-185.

SCHOEB, T.R.; MORTON, R. 1978. Scrotal and testicular changes in canine brucellosis: a case report. J. Am. Vet. Medicine Assoc. 172:598-600.
SERIKAWA, T.; KONDO, Y.; TAKADA, H.; YAMADA, J. 1984. Head-to-head type auto-spermagglutination with IgA antibody to acrosome induced by Brucella canis infection. Japan. J.I Vet. Sci. 46:40-48.

SHIN, S.J.; CARMICHAEL, L.E. 1999. Canine brucelosis caused by Brucella canis. En: Carmichael, L. (Ed.), Recent Advances in Canine Infectious Diseases. International Veterinary Information Service, Ithaca, NY. Disponible desde internet en: http://www.ivis.org (con acceso10/07/08).

WANKE, M.M. 2004. Canine brucellosis. Animal Reproduction Science (Paises Bajos) 82-83:195207.

ZAMORA, J.; ALONSO, O.; MARTIN, R. 1980. Brucelosis canina en Valdivia Chile. Estudio serológico y bacteriológico en perros de ciudad. Zentralbl. Veterinar Med. (Alemania). 27:149-153.

Recibido: Octubre 8 de 2008

Aceptado: Marzo 31 de 2009 